\title{
El método 7.1, Re-acción en Multi-re-forma: Re-generación Ética en la Tecnología Digital (TD) + Diseño Arquitectónico (DA)
}

\author{
Method 7.1, Re-action in Multi-re-form: Ethical Re-generation of Digital Technology (DT) + \\ Architectural Design (AD)
}

\author{
Dra. Gabriela I. Bustos, Arq. \\ Universidad del Zulia, Venezuela \\ bustosgabriela@gmail.com
}

\begin{abstract}
The goal of this article is to conceptualize and structure a theoretical proposed framed of ethics in digital technology in architectural design. The methodological vision of this proposal is one of complex epistemology, which seeks to understand applied ethics in a multicultural reality and the digital processes that reformulate contemporary architectural vision. The proposed "Method 7.1: Re-action in Multi-re-form" is based on Edgar Morin's "Method 6, Ethics", and Victor Martin's "Applied Ethics." The Method 7.1 is formed by seven ethical dimensions that constitute a pattern of complex thought that systematizes the values for the production of techno-architecture.
\end{abstract}

Keywords: Complex Ethics; Digital Technology (DT); Architectural Design (AD); Re-action; Multi-reform.

\section{Introducción}

Partiendo de los conceptos de ética en la complejidad en el Método 6, Ética, en Morín, (2006) y de Ética Aplicada en Martin,

(2008), se busca la aproximación conceptual a una ética arquitectónica desde una visión digital con responsabilidad planetaria. Mediante la propuesta del Método 7.1 se proyecta una ética compleja, que se enfoca en enriquecer una suma multidisciplinaria del Diseño Arquitectónico (DA) + Tecnología Digital (TD), con la concientización de su impacto transdisciplinar y de la importancia del habitante del espacio como consumidor y participe del producto creativo.

\section{Ética Aplicada Contemporánea}

\section{Cambio de percepción de la ciencia como emergencia ética.}

En su trabajo "Ética de la Ciencia", publicado en el 2008, Víctor Martin Fiorino deja claro el protagonismo del fenómeno tecno científico contemporáneo como determinador de estilos de vida que condiciona maneras de actuar, conocer y valorar.

Martin señala que la contemporaneidad está compuesta por una relación antagónica y vinculante entre poder-problemas-valores, que lleva a construir del bien personal al bien común, de una inteligencia de intervención a una inteligencia de integración. Indica que la reflexión sobre ética de la ciencia significa una propuesta seria de pensamiento crítico sobre el sentido de la actividad científica y sobre la magnitud del impacto de sus resultados.
En este sentido, entran en el discurso los entes o individuos afectados por los resultados de la actividad científica como participantes activos ante el proceso de producción.

En Martin el cambio de percepción de la ciencia surge como una emergencia a nuevos paradigmas que afectan tanto a la inteligencia científica como a la inteligencia ética:..."la inteligencia ética que se apoya en la teoría de las inteligencias múltiples y profundiza las propuesta de la inteligencia emocional y de la inteligencia social, se nutre de la capacidad de cambio y aprendizaje y de los mecanismos de trabajos derivados de las nociones de trasnculturalidad..." (Martin, 2008, p. 15).

Martin define inteligencia ética dentro de la capacidad de dar congruencia las acciones humanas en sus diferentes contextos, y en especial en cuanto a las acciones en la dimensión tecnocientífica: “...en particular en la dimensión tecnocientífica, se nutre de fuerza pedagógica que acompaña a lo que socialmente es valorado de manera positiva y considerado como deseable, como un bien que hay que alcanzar de manera inteligente, a través de la acción de una comunidad que impulsa una moral de los ciudadanos" (Martin, 2008, p. 15).

En la vía de cambio de percepción de la ciencia contemporánea, Martin identifica tres inclusiones importantes en el contexto y desarrollo del pensamiento humano:

La inclusión de la vida: se trata de reubicar el aporte del hombre en el marco de una ética de la responsabilidad como traductor y administrador de la vida pero no como su dueño. 
La inclusión en la complejidad: valorando la diversidad, pluralismo y tolerancia.

La inclusión en la realidad humana existente concreta: mediante el paradigma humanista, que recupera el conocimiento científico como obra de los seres humanos concretos. (Martin, 2008)

En Martín en la identificación de la emergencia de los paradigmas de inclusión, la ciencia y la ética se desarrollan desde dentro de la producción científica, mediante equipos de trabajos, y desde afuera, desde las exigencias de grupos sociales, generando cambios profundos que llevan al desarrollo de ideas de supervivencia, insertados en un sistema de vida responsable que busca orientar el conocimiento hacia problemas identificados como prioritarios. La importancia de la integración de la ciencia y la ética resulta en una vía de re-generación de un sistema de vida acorde con las exigencias de la sociedad compleja contemporánea:

"En la ética los cambios que el impacto de la explosión tecnocientífica ha impulsado se reflejan en una nueva valoración de la vida, hacia una inteligencia integradora y posibilitante, como instancia dralógica, de la convivencia en la diversidad. La convergencia entre ciencia y ética podría ser expresada en un recorrido que va del conocimiento al saber y del saber a la sabiduría." (Martin, 2008, p. 17)

\section{Ética en la Complejidad del Diseño con TD como Vía Re-generadora}

La concepción de una posición de generación y evaluación de diseño arquitectónico desde la ética compleja parece en sí misma una contradicción, una hipótesis inmedible colmada de incertidumbres y paradojas. ¿Cómo evaluar lo que por condición deber ser bueno? ¿Cómo establecer criterios para ello en una realidad compleja? ¿Cómo medir lo que siempre se está transformando? Parece como pretender atrapar un rápido pez con solo una intensión mental.

En este punto de la investigación, más que un recetario de acciones se busca plantear una visión de opciones para canalizar esa intensión de manera versátil, viable y posible. Si hablamos de producción tecnocientífica, y por consecuencia de ética de la tecnociencia, el asunto de la pertinencia, innovación, impacto humano-ambiental, relaciones humanas, comunicación, son solo algunos de los aspectos más resaltantes a incluir y vincular, o mejor, desde el discurso del Método 6, a re-ligar.

Sin embargo, no solo se trata de religarse con esta responsabilidad, o auto-ética o responsabilidad planetaria, esto es mucho más profundo y complejo.

Partiendo de la visión de Martin (2008), y refiriéndonos a la inteligencia ética, en este caso, inteligencia ética en la TD+DA, la creación del camino al compromiso, más que social, como civilización humana, es inminente en la actividad tecnocientífica del diseñador.

Cuando Martin (2008) se refiere a la emergencia ética de nuevos paradigmas, basada en una ética de inteligencias múltiples y profundas, la realidad apunta a los creativos a asumir el diseño como una vía para la generación de soluciones de vida que van más allá de la producción de accesorios digitales cotidianos. Se trata de generar sistemas de vida, que con apoyo en la TD, puedan brindar a los habitantes de los espacios íntimos y públicos, un contexto, un refugio propio para la convivencia compleja, múltiple, plural, diversa del ser cyber-social contemporáneo, incluyendo aquellos estratos sociales marginados de la vivencia tecnológica y aun peor, en situaciones de catástrofes naturales.

Para el desarrollo de una vía hacia para la ética aplicada y compleja en la TD+DA, se propone el Método 7.1, en honor a Morín y como consecuencia de su obra, Método 6, Ética. No se puede determinar solo el numero 7, pues la transformación constante de los procesos de re-forma ética va generando infinidad de versiones. Se trata de la primera versión de un enfoque optimista que posibilita una medida de dimensiones para la valoración del proceso y producto de diseño desde una regeneración ética de la TD+DA.

\section{Propuesta}

\section{El método 7.1: Re-acción en Multi-re-forma}

"El método adecuado es un desorden cuidadoso" Herman Melville. Moby Dick

En esta propuesta se establece una re-acción, como ecología de la inter-acción constante entre contexto, diseñadores y habitadores de los diseños (co-productores y consumidores del fruto tecnocientífico), contenida en un espiral de múltiples re-formas, entendiendo reformas como aquello que nos transforma, y como aquello que nos permite una reformulación de la "forma" de ser, entender y diseñar nuestro entorno. En este sentido se propone el método 7.1 re-acción en multi-re-forma.

Para el tratamiento de este método, se establecen 7 dimensiones de una ética compleja del diseño arquitectónico con TD:

1. Re-valoración - auto ética

2. Re-flexión - oportunidad en la crisis de la incertidumbre y contradicción

3. Inter-acción, ecología de la acción

4. Auto-organización

5. Trans-formación -en Morín mutación, en Martín con sensibilidad-

6. Re-Visión

7. Re-generación

Re-valoración - auto ética: El prefijo "re" se mantiene en la propuesta, en el significado de "hacer de nuevo, con otra visión" y de "repetición cíclica". En este sentido, cuando se habla de re- 
valoración, se enfoca en la búsqueda de un hacer de nuevo y en repetición cíclica un esquema de valores que permita la evaluación continua de las acciones tecnocientíficas, en este caso en particular de tecno-arquitectura. Esta re-valoración está dirigida a la reflexión sobre la importancia, rol, desempeño, trascendencia de las acciones realizadas, pertenencia de las acciones a realizar, impacto ambiental-humano, vigencia e innovación.

La re-valoración no puede ser solo de los factores externos de la producción tecno-arquitectónica. El diseñador o actor del producto debe estar sometido a una re-valoración constante, y establecer posiciones de auto ética que permitan su propia evolución como individuo conformador de una pluralidad social.

Re-flexión - oportunidad en la crisis de la incertidumbre y contradicción: re-flexión en tanto a: meditación y juicio continúo sobre la producción tecno-arquitectónica contemporánea en los diferentes escenarios de impacto. También se plantea la re-flexión en cuanto a "flexibilidad" o adaptabilidad en tiempo y espacio como condición de propuestas mutables, interactivas, elásticas, inteligentes de la producción tecno-arquitectónica. La adaptabilidad es una de las múltiples maneras de hacer una oportunidad en la crisis de la incertidumbre y contradicción contemporánea.

Inter-acción, ecología de la acción: Se plantea una acción en reproducción continua e interactiva entre el diseñador, el producto y los consumidores. Cuando Morín (2006) habla de la ecología de la acción dentro de las condiciones específicas donde se desarrolla el acto, Morín establece el contexto del acto dentro de la incertidumbre y contradicción ética como condiciones que determinan la relación entre intensión-acción-consecuencias. Al ciclo de relación interactiva de producción tecno-arquitectónica en un contexto complejo contemporáneo denominaremos, "interacción como ecología o reciclaje de la acción”, y sus influencias, consecuencias e impacto en el contexto donde se realice. En la complejidad la ley de interacción también está comprendida cuando la singularidad está inscrita en el universo y se combina el reconocimiento de lo local con la explicación de universo. En este sentido hace presencia un término muy usado en la red de la transdisciplina arquitectónica: el contexto.

Auto-organización: En el pensamiento complejo se ve la vida como un fenómeno de auto-eco-organización extraordinariamente complejo y que produce autonomía. En la auto-eco-organización de debe afrontar la dificultad de lo entramado como el juego infinito de inter-retroacciones, la solidaridad de los fenómenos entre sí, la bruma la incertidumbre y la contradicción.

“...Auto-eco-organización en el diseño arquitectónico: donde la autonomía de los eventos componentes de los micro sistemas y sistemas arquitectónicos son a la vez inseparables y dependientes de macro sistemas de inserción. Aquí los componentes y sistemas arquitectónicos se enlazan e interactúan de manera indispensable para su existencia, pero no con ello anulan su individualidad". (Bustos, 2009, p 191-192)

En la consideración de la auto-organización en la ética de la TD+DA, también se da la interdependencia entre el ser auto ético del diseñador, el impacto del objeto diseñado, el tiempo y espacio manejado, así como el contexto multidimensional del creador y de lo creado, cada una de estas instancias poseen una marcada autodefinición y singularidad que requieren la tolerancia, pertinencia, sensibilidad y eficiencia como valores éticos.

Trans-formación: Para Morín el camino de las reformas conduce a la metamorfosis, con ello a la ética compleja. En Martin este proceso de cambio debe realizarse con especial prudencia y sensibilidad. Para la concepción de una ética compleja en la TD+DA, se plantea aun camino de trans-formación, que implica un sistema de cambios mediante multi-re-formas de la visión, producción, pertinencia, viabilidad e impacto de la tecnoarquitectura. La trans-formación, no pretende mutación, se busca un proceso suave de generación de estructuras de pensamiento que vayan construyendo nuevos paradigmas de existencia humana y por consecuencia de construcción de su propio hábitat.

El "trans" implica en sí mismo un espacio de transición, un "entre" que contiene el margen reflexivo para detenerse a estudiar la "formación" del ser ético del productor de tecno-arquitectura, la "formación", que es importante y urgente que este productor en potencia reciba en su proceso académico, y la "forma" como enfocar y viabilizar esta "formación" ética compleja.

Re-visión: La re- visión como estrategia de evaluación en la ética en la TD+DA se enfoca de doble manera: la primera como la necesidad de reponer, restablecer los esquemas de valores y responsabilidades planetarias como parte fundamental de la formación del diseñador. La necesidad de restituir en el proceso de diseño la trascendencia el rol del productor de tecnoarquitectura y la predicción o visualización de los posibles impactos de su producto.

El segundo enfoque de re-visión en tanto a nueva posición de objetivos, se centra en un asunto de pertinencia, espacio y tiempo. Para este enfoque se asume como principal aporte teórico el realizado por Machado 2012:

"Se utiliza la posición como sustento para la TRANSposición, como concepto que agrupa múltiples significados que conectan: el medio físico, geográfico, intelectual, experimental, experiencial y contextual de la arquitectura, para establecer el modo y el territorio que opera, como ancla hacia un despliegue de lo que involucra, como campo de una perspectiva en la arquitectura". (Machado, 2012, p 32)

El asunto de la posición, en Machado desde la trans-posición, es una visión fundamental de internalizar tanto en el proceso creativo de tecno-arquitectura, como en su sistema de valores para su evaluación y sentido ético.

Re-generación: La re-generación ética, implica la religación de las otras 6 visiones anteriores: re-valoración + re-flexión + interacción + auto-organización + trans-formación + re-visión, como base para un sistema estratégico de evaluación desde la ética compleja en la TD+DA.

La re-generación, significa el nacimiento de lo nuevo, así como la sustitución de lo no tan bueno por lo mejor tanto dentro del esquema de valores de los procesos académicos de formación tecno-arquitectónica, como en el ámbito comercial o de "generación" del producto. Hay la apremiante necesidad de 
soluciones ingeniosas ante problemas complejos de diseño contemporáneo, donde la disciplina de la TD es un soporte tanto como sistema de vida y enfoque del desarrollo humano, como también asidero de posibilidades y viabilidad de innovación. El reto, es que dicha innovación sea aplicada éticamente en las comunidades demandantes de esas soluciones ingeniosas.

La ética compleja en la TD+DA, se ubica en la total post-revolución no solo digital, sino también integral de la arquitectura contemporánea, con sus implicaciones de diseño y construcción y las nuevas tecnologías, conformada por aquellos que han sido educados en ambientes digitales desde los inicios y que buscan un sentido más amplio de la aplicación de la TD en el diseño, explorando otras disciplinas y estableciendo argumentos tecnocientíficos, filosóficos y epistemológicos que fundamentan sus ideas.

La propuesta de estas 7 visiones hacia una ética en la TD+DA, son solo algunas de las vías para guiar un sistema que permite concebir y medir la producción de tecno-arquitectura desde una visión contemporánea compleja, hacia el asunto ético desde la dimensión integral de diseño como un camino para el desarrollo del hábitat humano.

\section{Conclusiones}

El planteamiento del método 7.1, y las sietes dimensiones éticas: re-valoración - auto-ética; re-flexión; inter-acción, ecología de la acción; auto-organización; trans-formación; re-visión; regeneración, constituyen un esquema de pensamiento complejo que sistematiza una base para sustentar valores de producción tecno-arquitectónica.

Una re-acción en multi-reforma es inminente para la autoregeneración de la producción tecno-arquitectónica contemporánea. La re-acción, que también puede ser entendida como inter-acción, desarrolla pluralidad de pensamiento, nociones filosóficas, técnicas y estratégicas de la producción ética tecnocientífica.

El Método 7.1 constituye un medio teórico-conceptual que establece dimensiones de medida para la valoración ética de producción arquitectónica. Como reto luego de esta propuesta, se plantea una segunda fase para el diseño de un medio donde se pueda poner de manifiesto este Método 7.1 de manera "prácticamente" medible, a través de redes sociales tridimensionales, trans-formando su impacto, como redes académicas y de práctica profesional para la generación de tecnoarquitectura, en sistemas multimediales.

Este es el marco de un discurso ético complejo en la arquitectura con TD:

Complejo pues es consecuencia de una mixtura de "materialidades" estratificada sobre intereses políticos, económicos, sociales y tecnocientíficos.

Se trata de una ética compleja en la arquitectura, pues es una y múltiple a la vez, es una en cuanto al alcance de un bien común planetario, y es múltiple en tanto a diversidad de dimensiones, actores, intereses y consecuencias.

Es compleja pues no está escapa a la incertidumbre contemporánea, la ética en la arquitectura con TD no puede tener una sola visión del mundo, cada fragmento social tiene necesidades de hábitat diferentes, por lo tanto las respuestas de diseño tienen que ser diversas y versátiles, aunque logrando el mismo fin.

La ética compleja en arquitectura desde la TD debe autoregenerarse constantemente, fundada en el "amor" de brindar al "otro" un refugio de vida, una urbanidad, un hábitat contenedor de memorias colectivas, físicas y digitales.

\section{Referencias}

Bustos, G. (2009). Tutor: Dr. Martin, Víctor. Epistemología compleja del diseño arquitectónico con Tecnología Digital: ASMI en los talleres de diseño arquitectónico. Trabajo para optar al título de Doctora en Arquitectura. División de Estudios para Graduados. Facultad de Arquitectura y Diseño. Universidad del Zulia.

Machado Penso, M. V. (2012). Desde la TRANSformación hacia la TRANSposición. Trabajo para optar al título de Doctora en Arquitectura. División de Estudios para Graduados. Facultad de Arquitectura y Diseño. Universidad del Zulia.

Martin Fiorino, V. R. (2008) Desafíos actuales de la Ética Aplicada. Uniojeda Fondo Editorial de la Universidad Alonso de Ojeda, Costa Oriental del Lago, Maracaibo, República Bolivariana de Venezuela.

Morin, E. (2006). El Método 6. Ética. Ediciones Cátedra (Grupo Anaya, S. A.), 2-6. Juan Ignacio Luca de Tena, 15. 28027 Madrid. 